\title{
A CLEC-based Study on the Spatial Metaphor of HIGH Collocations
}

\author{
Huiying Zhang \\ School of English Language, Culture and Literature, Beijing International Studies University, China \\ Weijie Zhou \\ School of English Language, Culture and Literature, Beijing International Studies University, China
}

\begin{abstract}
Metaphor is conceived as understanding and experiencing one kind of thing in terms of another. Metaphor is pervasive not just in language, but also in our thoughts and actions. The conceptual system is metaphoric in nature. Previous studies on metaphor are done through linguistic researchers' intuitive analysis. Few quantitative studies have been done to metaphor from corpus and the perspective of learners in second or foreign language learning. This paper, based on CLEC corpus, attempts to explore the relationship between spatial metaphor and second language acquisition and learning. Using 'HIGH' as a spatial measurement adjective, the paper hopes to understand different types of metaphorical collocations of HIGH in CLEC - a Chinese EFL learners' corpus, and to indicate the relationship of metaphor output ability with learners' language proficiency. The studies have demonstrated eight types of HIGH collocations from Chinese EFL learners and the metaphor output ability is directly related to the learners' language proficiency.
\end{abstract}

Index Terms-metaphor, conceptual metaphor theory, spatial metaphor, CLEC, HIGH collocations

\section{INTRODUCTION}

Metaphor is usually defined as "a figure of speech that, for rhetorical effect, directly refers to one thing by mentioning another" (http://www.merriam-webster.com/dictionary/metaphor). In other words, for most people, metaphor is an extraordinary rather than ordinary language in that it is a rhetorical device with the poetic imagination (Lakoff \& Johnson, 2003), so metaphor can be treated as a language alone, which has no connection with thought and action. However, Lakeoff \& Johnson (2003) believe that metaphor inherently exists in the conceptual system depicted through language, thought and action. Such a statement has thus greatly changed metaphor from its language level to a conceptual level and the birth of Conceptual Metaphor Theory (Lakoff, 1993) has broaden the studies in cognitive linguistics.

In metaphor research, most studies on metaphor are conducted based on researchers' intuitive analysis, and few quantitative studies have been done to understand how EFL learners are using metaphor in actual uses. In 1990s corpus was introduced into metaphoric studies (Xue\&Xiang, 2018) and was widely accepted as scientific by its huge amount of data and convenient information extraction. Therefore, corpus-based metaphorical studies (CBMS) were booming in the past several years. Until now, basing on corpora, some researchers have studied interlingual and Orientational Metaphor, like "UP" and "DOWN"(Liu \& Liu, 2006); interlingual and structural metaphor, like "grammar structure" (Wang, 2009); and there were also some works concerning about interlingual and Ontological Metaphor (Gong \& Zhou, 2009).

Some studies $(\mathrm{Xu}, 2014)$ have shown that a good understanding of metaphor may promote second language acquisition. This paper, through investigating CLEC corpus on Chinese EFL learners' uses of HIGH in different collocations, attempts to understand the learners' metaphor output abilities in spatial metaphor and explore from quantitative and qualitative aspects the uses of different collocations with HIGH.

This body of the paper unfolds in 4 parts. In the first part, the definition of Conceptual Metaphor Theory is introduced. In the second part, an analytic review is done on the development of CMBS; in the third part, CLEC corpus is used to explore the relationship between spatial metaphor and second language acquisition and learning. The research questions, the methods, and the criteria for the study of HIGH collocations are included in this part; in the fourth part, the results of the study are displayed and discussed.

\section{UnDERSTANDING CONCEPTUAL METAPHOR THEORY (CMT)}

According to Lakoff and Johnson (2003, p.5), "the essence of metaphor is understanding and experiencing one kind of thing in terms of another". They further assert that the conceptual system, which structures what one thinks, what one acts and relates to other people, is metaphoric in nature (2003). However, what should be treated with caution, according to Lakoff and Johnson(ibid.), is that one may sometimes think and act somewhat automatically along some patterns and such patterns are not quite obvious in terms of the conceptual system. Therefore, language, sharing the same conceptual system with thought and action, can be employed as an important source of evidence to understand the 
conceptual system.

In accordance with CMT (Lakoff \& Johnson, ibid.), metaphor can be classified into three parts: Structural Metaphor, Orientational Metaphor and Ontological Metaphor.

Structure Metaphor is one that is metaphorically structured in terms of another (Lakoff \& Johnson, ibid.). In the sentence of ARGUMENT IS WAR, the concept ARGUMENT can be referred to as a situation in which two or more people strongly disagree with each other. And WAR occurs when there is fighting between two or more countries or between opposing groups within a country, involving a large number of soldiers and weapons. So through mapping, that is, when we map WAR into the ARGUMENT, we are aware that participants are in different groups, who defend their own opinions and the argument result could be either to win or to lose.

Ontological Metaphor contained two parts: Container Metaphor and Entity and Substance Metaphor. In Container Metaphor, the surface is equal to the target domain and its content means source domain. Lakoff and Johnson(ibid.) claim that the content is highlighted with respect to its amount, density, centrality, and boundaries. Entity and Substance Metaphor usually regard abstract ideas as entity, so Entity and Substance Metaphor can fulfill the following purposes: referring, quantifying, identifying aspects, identifying causes, setting goals and motivating actions (Lakoff \& Johnson, ibid.).For example, the sentence LIFE IS A JOURNEY is a typical Container Metaphor. Another typical example is RAISING PRICE. Generally, only physical things can be moved or lifted to a higher position or level. PRICE cannot be seen, nor can it be touched. It is an abstract concept. By RAISING PRICE, people can consciously compare the former and the current price, then find out the reason of raising, and take some actions.

Orientational Metaphor organizes a whole system of concepts with respect to one another (Lakoff \& Johnson, ibid.). Based on Orientational Metaphors, a concept of Spatial orientation emerges, which refers to using physical direction to organize another concept, like, UP/DOWN, HIGH/LOW, and so on. Lakoff and Johnson (1980, p. 15) have used spatial metaphor to explain how UP and DOWN have organized another concept. In the concept of HAPPY IS UP; SAD IS DOWN, such expression as "I'm feeling up; That boosted my spirits; My spirits rose; You're in high spirits" are often used to express the happy feelings. In our daily life, an erecting posture generally indicates a positive emotional state. With such an image map adding to human's emotion, positive words are related to happy and bright feelings. On the contrary, when people say "I'm feeling down; I'm depressed; He's really low these days; I fell into a depression; My spirits sank", (ibid.) the downward words are used to express the sadness as drooping postures typically go along with sadness and depression. Besides, CMT has included many other interesting Spatial Metaphor hypothesis, like such sentences as: MORE IS UP, LESS IS DOWN; GOOD IS UP, BAD IS DOWN; HIGH STATUS IS UP, LOW IS DOWN; HEALTH AND LIFE ARE UP, SICKNESS AND DEARH ARE DOWN, and so on.

Lakoff and Johnson's Conceptual Metaphor Theory(CMT) (2003) recognizes five tenets of conceptual, that is, 1) Metaphors structure thinking; 2) Metaphors structure knowledge; 3) Metaphor is central to abstract language; 4) Metaphor is grounded in physical experience; and 5) Metaphor is ideological.

\section{The DeVelopment of CoRpus-BASEd Metaphor Studies (CMBS)}

Corpus has promoted the development of linguistics and formed a new linguistic branch, corpus linguistics. As a relatively scientific research tool, it was booming in 1980s. According to Alice (2005), corpus was a collection of linguistic data held in machine-readable form. And Wang (2012) defined it more comprehensively as real and authentic materials in a large scale, being stored in and supported by computer technology, following linguistic rules.

Corpus, in addition to the significant storage capacity of huge amounts of message, can perform retrieval with the aid of computer technology. So far researchers have built up various corpus databases to meet different research objects, like the earliest corpora Brown corpus, constantly updated; the large scale English native speakers' corpus, such as BNC and COCA (researched and developed by Lancaster University); Chinese native speakers' corpus CCL; Corpus of specific purpose: Yang's Corpus-based Analysis of Chinese Learner English (CLEC); Wen and Wang's Spoken and Written English Corpus of Chinese Learners (SWECCL), and so on. Corpus development, together with the development of studies from other linguistic fields, has increased human understanding of language.

Corpus has also boosted the studies of metaphor. Since previous studies of metaphor are based on researchers' intuitive analysis on language, CMT studies, via a corpus-based approach, has proved correctness of CMT with corpus (Wu, Zhang, et. al. 2016) and shed new light on the empirical and social aspects of cognitive linguistics (Sun, 2012). In addition, the combination of the two can provide a quantitative approach to study EFL learners' metaphor use. Wang (2015) studies Chinese EFL learners' verb collocations and has found that L1 may have a negative transfer to L2's noun and verb acquisition. Chen (2018) comes to a similar conclusion in her study and she further points out that Chinese EFL learners' metaphor use is limited in type and quantity. Besides, Shi and Tang (2018) have also found that in terms of high frequency metaphor, Chinese EFL learners have displayed similar performances as those of native speakers; but they are low in frequency of using metaphor. Besides, filtrated methods have also enriched corpus studies, for example, by Antconc, Wordsmith, Wmatrix as well as the development of CMT and SLA.

Up to now, previous studies on spatial metaphor have made some achievements, but only a few of them focus on High and its collocations. After studying BNC corpus, Zhou and Zhang (2003) found that HIGH and LOW mainly had five target domains: quantity, hierarchy, condition, sense and time. By studying the entitative collocation of HIGH in English and Chinese, Chen (2010) found that the collocation of HIGH had many common points in the field of high 
frequency collocations, but it had great differences in marginal collocations. Similarly, Liu (2012) found that Chinese and English had many common points in the aspect of HIGH collocation, but the culture, history and cognition contribute to the differences in creating the collocations. Those studies have promoted the understanding on HIGH and its metaphors. As there are only a few researches, the metaphorical study on HIGH and its collocation is still very necessary.

\section{A CLEC- CORPUS STUDY ON HIGH COLLOCATIONS}

Corpus-based metaphor studies in the field of foreign language teaching not only can increase the understanding of the quantity and quality of metaphor use from Chinese ESL learners, but also can help the learners to raise their awareness in building up a well-designed metaphorical system in English language learning, thus improving their language proficiency.

According to Wienold and Rohmer (1997), the relationship within spatial measurement adjectives is that size < length < distance < depth < height. In this study, the author of this paper takes $\mathrm{HIGH}^{*}$ as an example to investigate two questions:

1) In Chinese EFL learners' corpus, how many metaphorical types of HIGH are there?

2) Is metaphor output ability closely related to their current language proficiency?

\section{A. The Instrument of the Study}

Corpus-based Analysis of Chinese Learner English (shortened as CLEC)(Yang, Gui \& Yang 2005), is one of the earliest learners' writing test corpora to study Chinese EFL learners' English learning. It contains five sub-corpora: St2 collects high school students' composition; St3 is the composition of College English Test Band 4(CET4); St4 is the writing test of texts of College English Test Band 6(CET6); St5 contains daily compositions of freshman and sophomore English-major students. And writers in St6 are better at English as they are junior and senior English- major students. The corpus totally has a million words, and each sub-corpus has 200 thousand words.

Although CLEC is famous for its authentic materials, various topics, clear classifications and huge amount of storage capacity (Yang, 2005), it is only a huge database. To deal with the corpus, the author has chosen Antconc, developed by Laurence Anthony (2011) to analyze and study monolingual data, as the retrieval tool. Antconc is chosen because it has many functions, like Concordance, Concordance Plot, File View, Clusters, N-Gram, Collocate, Word List and Keyword List. It is a simple but efficient tool.

Metaphor recognition is a key issue in corpus-based metaphor research and some standard recognition criteria are important.

Metaphor Identification Procedure (MIP) was firstly proposed by Pragglejaz Group (2007). Therewith, this group has updated the criteria and developed Metaphor Identification Procedure \& Vehicle Identification procedure(MIPVU). The two procedures agree that a metaphor is generated when its basic meaning is different from its contextual meaning. This paper has chosen MIP as the criteria.

According to Pragglejaz Group (2007), the MIP works in four steps.

First, any lexical unit in a text is examined based on the context, that is, what comes before and after the lexical unit; second, other meanings of the lexical unit should be found in other contexts, e.g. from the dictionaries. The criteria for the basic meanings are that they are not necessarily the most frequent meanings of the lexical unit; they can be more concrete [what they evoke is easier to imagine, see, hear, feel, smell, and taste], related to bodily action, more precise (as opposed to vague), and historically older. Third, if the lexical unit has more basic meanings in other contexts than the given context, we should decide whether the contextual meaning of the lexical unit is different. If there is any difference, the fourth step is to mark the lexical unit as metaphorical.

\section{B. The Research Procedure}

This paper takes CLEC as the target corpus and Antconc as the retrieval tool, following MIP to resolve two research questions: Chinese EFL learners' metaphorical production and the relevance between the production and their actual language proficiency.

To solve Question 1, this paper has adopted the following four steps. First, uploading the whole CLEC to Antconc and making a retrieval of "high*" (* means high and its variants), and cloning the retrieval data to the Microsoft Program of Excel. Next, following MIP to cancel original meaning and picking out metaphor collocates, which appear at least ten times. Then, according to their metaphorical meaning, dividing them into different groups and tabulating the collected data into Table 1. At last, drawing conclusions of Question 1 through the table.

And to answer Question 2, the author has used the filtration function of Excel to reprocess Table 1 by separating it according to sub-corpus and set up each one's own list to make comparisons. Typical expressions are picked out to answer the question.

\section{FINDINGS AND DISCUSSION}

Studies (Xu, 2014) have shown that a good understanding of metaphor can help students develop and acquire the conceptual thinking and action through the learning of the target language. This paper has taken HIGH and its 
collocations as an entry point, analyzing the writing tasks in CLEC and then studying them from the perspective of Spatial Metaphor. This paper has found that Chinese EFL learners are equipped with the metaphor output ability.

\section{A. Understanding Varieties of Metaphorical Types of HIGH in Chinese EFL Learners' Corpus}

Table 1 is the results of Question 1. It can be found that there are nine types of metaphor of HIGH and its collocations. Sorted from more to less, they are: quantity, hierarchy, quality, speed, time, degree, emotion, and sense. Each type has no less than two subsets, besides emotion. Table 1 only displays high frequency metaphorical meaning, and the low frequency collocations, the collocations which are less than 10 are not taken into account.

To clearly explain those metaphorical meanings, the author randomly picks out 16 typical examples (the sequence number of each example is corresponding to the sequence number in Table 1):

E.g.

1. If a man or woman gets a high salary, he or she will lead a comfortable life. (St6) (number)

2. Now I have graduated from the college. I get high scores in all my subjects, especially in English. (St5) (rate)

The principle of QUANTITY metaphor (Example 1 and Example 2) is that HIGH means more while LOW means less. It is oriented from learners' experience of height. If a children(A) is $150 \mathrm{~cm}$ and his friend(B) is $160 \mathrm{~cm}, \mathrm{~B}$ is taller than A from the visual comparison. And such experience is mapped into learners' cognition that $160 \mathrm{~cm}$ is higher than $150 \mathrm{~cm}$. Whether in number or rate, SALARY or SCORE, can be represented with number in certain condition, and if a number is more than another, students think it is higher. Therefore, HIGH means more in this situation.

3. All modern wars need high technology, and high technology needs professional soldiers. (St6)(advanced)

4. But if you are in a higher position, and I could do more skillful job (St 6).(status)

The HIERARCHY metaphor is quite similar to Lakoff and Johnson's view (2003) that HIGH STATUS IS UP; LOW STATUS IS DOWN. And such metaphor can be explained from learners' knowledge of social class. In slavery society and feudal society, people were divided into several classes.

While a triangle can be used to explain such relationship among people, the author of this paper is more inclined to employ the concept of mountain. Upper class is considered similar to the top of the mountain. They are built over the underclass. At the same time, they enjoy more priorities than the underclass. With such experience mapped into people's mind, people have also classified matters into different classes. That is, after dividing the same things into different classes, if one of them is quite excellent, he enjoys a higher rank. And in Example 3 and Example 4, HIGH TECHONOLOGY and HIGH STATUS are working in this way.

5. In a highly developed city to find a good job is very difficult. (St4) (well)

6. The people's living standards are higher and higher. (comfortable)

The principle of QUALITY metaphor can be explained by Lan's statement of physical health. Lan (1999) claims that HIGH is closely connected with the physical health, because healthy people could stand straightly and feel comfortable, while unhealthy people would usually lie down on the bed or even died. Therefore, people have formed an image that being good or comfortable is HIGH. And that's why in Example 5 and Example 6, learners describe good things and comfortable feelings as HIGH.

7. So, the combat effectiveness will not be high and their will to fight will not be firm. (efficiency)

8. China is developing in a high speed. (quick)

The SPEED metaphor means that rapidity equals to HIGH. Such mapping can be traced back to ancient China. At that time, tools were simple and rare. And stature could be a favorable factor. Generally speaking, a tall person could do more things than short ones. For example, if a tall person and a short person go out to find food, there is no doubt that the taller ones can pick up fruit in higher place and harvest more food at the same time. So learners map such images into speed. If one thing is quick or efficient, we'd like to describe as HIGH, just like the Example 7 and Example 9.

9. It is high time for us to deal with this problem. (st4,)(top)

10. The body is strong, so the life expectancy is high(St3) (long)

Besides the mapping which comes from human experience, natural experience can also influence our concept. A typical example is that the solar activity has greatly shaped the TIME metaphor. In ancient time, light was a very important resource for people to do housework or farm work. Their life was greatly affected by the sun. Without advanced technology and science, ancient people had such misunderstanding that in the morning, the sun was the highest, because the temperature was lower than the noon. Such situation was suitable to do housework or farm work. That's why people use high time to express a thing that is urgent to do. However, in Example 11, learners' usage of LIFE EXPECTANCY IS HIGH is transferred from Chinese way of thinking "gaoshou", which refers to a phenomenon that a person lives quite a long life. After searching in COCA, the author found that the co-occurrence of LIFE EXPECTANCY and HIGH's frequency is only ten times. And native speakers usually use INCREASE to describe such phenomenon. The relationship between language and cultural norms has indicated that metaphorical thinking is also greatly influenced by people's cultural background. 
Table 1 HIGH and its collocations' metaphor types in CLEC

\begin{tabular}{llll}
\hline Types & Metaphorical meaning & Typical examples & Percentage \\
quantity & 1. number & e.g. high score/salary & $35.14 \%$ \\
& 2. rate & e.g. high infant mortality & \\
hierarchy & 3. advanced & e.g. high technology/education & $27.82 \%$ \\
& 4. status & e.g. high social position & \\
quality & 5. Well/good & e.g. high developed city/ high & $10.33 \%$ \\
& & intelligence & \\
speed & 6. comfortable & e.g. high living condition & \\
& 7. efficiency & e.g. the combat effectiveness & $9.70 \%$ \\
time & 8. quick & 9. top & e.g. economy increase in high speed \\
& & e.g. high time to deal with the & $5.09 \%$ \\
& 10. long & important social problem & \\
degree & 11. big & e.g. higher life expectancy & \\
& 12. Heavy/important & e.g. high pressure & $3.97 \%$ \\
emotion & 13. happy & e.g. high spirit & \\
sense & 14. touch & e.g. high temperature & $2.70 \%$ \\
& 15. hear & e.g. sing highly & $2.38 \%$ \\
\hline
\end{tabular}

11. People live in high pressure(St5). (big)

12. The problem is that they think too highly of the society. (St6)(heavy)

The formation of DEGREE metaphor can be explained from people's recognition about big objects. Take a square block of wood as an example. With the same length and width, the higher one must be larger in volume. Such imagination was mapping to abstract matters. If people think an abstract matter that is difficult, hard, and important, they may form an image of a high object, just like Example 11 and Example 12.

13. and we are all in high spirits. (St2) (happy)

When we explain the EMOTION metaphor, Lakoff and Johnson's theory (2003) is very helpful. They claimed that drooping posture typically goes along with sadness and depression, while an erecting posture goes with a positive emotional state. When a person is happy, he may hold his head high and his mouth is upward.

14. If you throw the frog into the water with high temperature, the frog will jump at once(St4). (touch)

15. But they didn't say that couldn't see the cloth and sing highly praise for the cloth. (hearing)

The SENSOR metaphor in CLEC mainly contains two types: HEARING and TOUCH. Zhou and Zhang (2003) have also paid attention to this phenomenon and they think the sound BIG or SMALL is determined by people's figures. A taller person usually is stronger and can phonate loudly, which can be used to explain Example 15. The reason of high temperature is indirectly mapping from quantity. The hotter it is, the bigger is the number in the thermometer. When people feel hot, they are consciously aware that the temperature number is increased.

\section{B. Understanding the Relationship between Metaphor Output Ability and the learners' Language Proficiency}

This paper has also found that language proficiency is closely related to metaphor output ability. English major and higher level students are equipped with more metaphorical expressions with HIGH. From Table 1, QUANTITY is the most frequently used metaphor of HIGH, so the author will further explore its collocations by analyzing their subsets. 


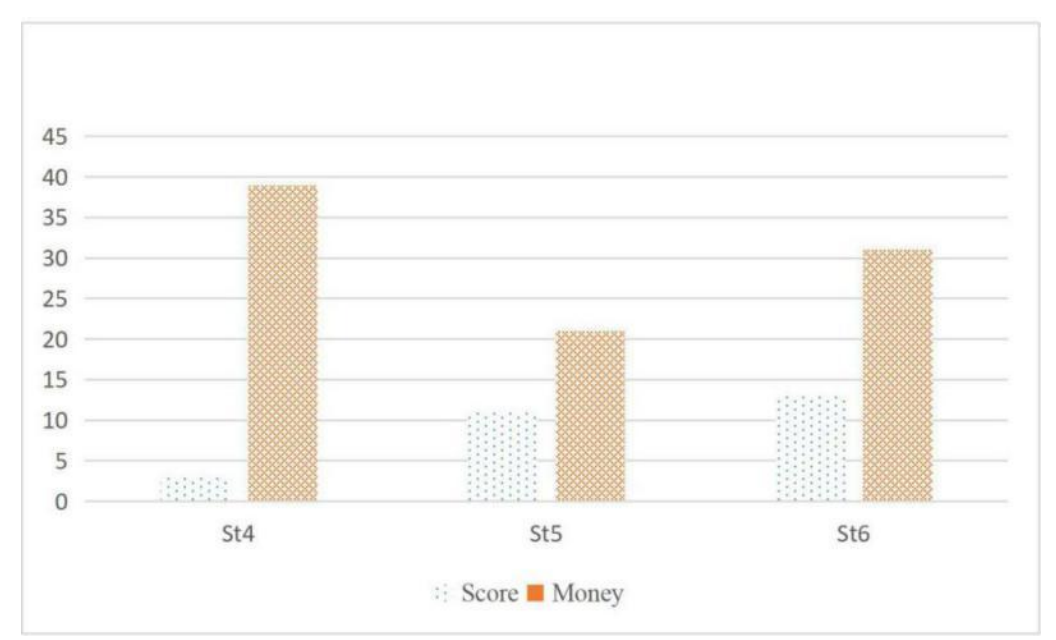

Figure 1 The usage of score and money with HIGH in ST 4-6

In the concept of number (Figure 1), there are two major topics: money and score. It is because people's experience can greatly affect their thinking methods and their topic selections. Score is a common topic in all these five sub-corpus, and it is more common in middle and low level students' compositions. However, university students, especially those with high grades, are under more pressure from their future careers. Among St4, St5 and St6, money is more frequently used.

From Table 2, we can draw the conclusion that whether in incoming or outgoing aspects, the used expressions of ST6 are more than ST4 and ST5. As St4 refers to junior and senior non-English majors, St5 freshman and sophomore of English- major students, and St6 junior and senior English majors, the language proficiency ST6 is higher than ST4 and ST5, which corresponds to the results - metaphor output ability is directly relevant to the leaners' proficiency.

Table 2: the expressions of MONEY in ST 4-6

\begin{tabular}{ll}
\hline level & \multicolumn{1}{c}{ Expression of money } \\
\hline ST4 & Incoming: high profit; high income; charge high; high reward; high pay; \\
& high earning; high salary; high expenses; high wages \\
\cline { 2 - 2 } & Outgoing: high cost; pay high money; high price \\
\hline ST5 & Incoming: high cost; high income; high-paid job; high salary; high \\
& payment; \\
\cline { 2 - 2 } ST6 & Outgoing: high price; high tuition and expenses \\
& salary; high award; high profit; \\
\cline { 2 - 2 } & Outgoing: high cost; high medical expenditure; high expenses; high \\
medicine fees
\end{tabular}

The result of this paper is quite similar to the study from Zhou and Zhang (2003), who studied HIGH and LOW by studying BNC, a large scale native English speaker corpus. They divided their results into five types (from more to less): quantity, hierarchy, condition (emotion \& quality), sense (hearing, smell, visual) and time. As their paper studied two dimensions, the author has picked out some relevance information and data, and tabulated a new table (Table 3 ). Both of this paper and Zhou and Zhang's result (2003) showed that from HIGH collocations used by Chinese EFL learners and English native speakers, quantity, hierarchy, and quality have shown high-frequency in Spatial Metaphor.

Certainly there are some differences in the two studies in that that Chinese EFL learners have frequently used TIME metaphor of HIGH, but they seldom use SENSE metaphor. However, native English speakers are just the reverse. It proved Lakoff and Johnson's opinion(2003:20) that "our physical and cultural experience provides many possible bases for spatialization metaphors. Which ones are chosen, and which ones are major, may vary from culture to culture". The study has confirmed that cultural perception, as an important part in conceptual system, may have positive or negative impact in the interlanguage phase in Chinese English Speakers. 
TABLE 3

THE METAPHOR STUDY OF HIGH IN BNC (FROM ZHOU AND ZHANG, 2003)

\begin{tabular}{lll}
\hline Types & Metaphor & Percentage \\
\hline \multirow{2}{*}{ quantity } & Number & $20.0 \%$ \\
\cline { 2 - 3 } & Degree or density & $26.1 \%$ \\
\hline hierarchy & Social status & $5.5 \%$ \\
\cline { 2 - 3 } & General hierarchy & $11.7 \%$ \\
\hline Condition & Quality & $8.4 \%$ \\
\cline { 2 - 3 } & Emotion & $3.5 \%$ \\
\hline sense & listening & $1.9 \%$ \\
\cline { 2 - 3 } & smelling & $0.1 \%$ \\
\cline { 2 - 3 } & Visual sense & $0.1 \%$ \\
\hline Time & & $1.1 \%$ \\
\hline
\end{tabular}

\section{CONCLUSION}

This paper, through CLEC corpus, has studied the Spatial Metaphor of HIGH collocations from Chinese EFL learners. Incorporating Lakoff and Johnson (2003)'s Spatial Metaphor theory, this study has shown that Chinese EFL learners have used eight types of metaphors with HIGH and its collocates in their writing, and the metaphoric expressions are closely related to the learners' language proficiency. The higher the learners' English proficiency is, the more metaphors the learners can produce. In addition, the results have also demonstrated the existence of interlanguage in Chinese EFL learners' English learning. The examples from CLEC-corpus have indicated that Chinese conceptual system may have some positive or negative influence on learners' English metaphor expressions. In fact, if two languages share some similar conceptual ideas, Chinese EFL learners' metaphor expressions are understandable; otherwise, the HIGH collocations may carry some Chinglish elements and may be confusing to English native speakers.

Therefore, in the future, more studies on metaphor uses from EFL learners should be done so that the research findings can be integrated to language teaching to raise learners' awareness of the different conceptual systems of different languages.

\section{REFERENCES}

[1] Alice, D. (2005). Metaphor and Corpus Linguistics. Philadelphia: John Benjamins Publishing Company.

[2] Cameron, M. \& Graham, L. (2001). Researching and Applying Metaphor. Shanghai: Shanghai Foreign Language Education Press.

[3] Chen, L. A. (2018). Corpus-based Metaphorical study on English Economic Discourse. Journal of PLA University of Foreign Languages. 04, 34-42.

[4] Chen S.T. (2010). A Comparative Study on Spatial Measurement Adjectives -- Take HIGH and TALL as Examples. Shandong Foreign Language Teaching Journal. 05, 14-20

[5] Chen, Y.R. (2016). A Corpus-Based Study on Metaphor Production of Chinese Learners of Second Language --Evidence From English Plant Metaphors. Journal of Guangxi University for Nationalities (Philosophy and Social Science Edition). 02, 185-190.

[6] Definition of METAPHOR Accessed May 27 $7^{\text {th }}, 2019$ from: http://www.merriam-webster.com/dictionary/metaphor.

[7] Gong, Y.M \& Zhou R. (2009). A Corpus-based Study on Ontological Metaphor--A Case Study of WIDE. Computer-Assisted Foreign Language Education in China. 01, 25-29.

[8] Lakoff, G. (1993). The Contemporary Theory of Metaphor. In R. Gibbs (ed.) Metaphor and Thought. New York: Cambridge University Press.

[9] Lakoff, G. \& Johnsen, M. (2003). Metaphors we live by. London: The university of Chicago press.

[10] Lan, C. (1999). ACognitive Approach to Spatial Metaphors in English and Chinese. Beijing: Foreign Language Teaching and Research Press.

[11] LaurenceAnthony. (2011). AntConc (Version 3.2.1) [Computer Software]. Tokyo, Japan: Waseda University. Available from http://www.laurenceanthony.net/software.

[12] Liu, K. \& Liu, H. (2006). The Contrastive Study on Spatial Word UP. Shandong Foreign Language Teaching Journal. 04, 4245.

[13] Liu, Y. (2012). Comparison of Cognitive Metaphors of English and Chinese Longitudinal Spatial Dimension Words -- Take HIGH/LOW as an Example. Journal of Kaifeng University. 01, 45-48.

[14] Liu, Z.G. (2016). The approach and metaphorical Teaching of Intercultural communicative competence in College Foreign language Teaching. Journal of Northeast Normal University (Philosophy and Social Sciences), 03, 121-124.

[15] Pragglejaz Group. (2007). MIP: A Method for Identifying Metaphorically Used Words in Discourse. Metaphor and symbol, 01, 1-39.

[16] Shi, J.F \&Tang, B. (2018). A study on the metaphorical semantic knowledge of L2 Learners: a Corpus-based Comparative study of Human polysemous words. Journal of Xi'an InternationalStudiesUniversity.03, 18-28.

[17] Sun, Y. A. (2012). Corpus-based Approach to Metaphor Use: A Case Study of H1N1 Flu Discourse in Chinese and American Media. Foreign Language Research, 164, 51-54.

[18] Wang, K.F. (2012). Corpus-Based Translation Studies. Shanghai: Shanghai Jiao Tong University Press.

[19] Wang, R. (2015). A Corpus-based Study on Verb-noun Collocation Errors by Chinese Non-English Majors from the Perspective of Conceptual Transfer. Foreign Language Research. 03, 120-124.

[20] Wang, R.Q. (2009). Grammatical Metaphor and the Entry of Self-designation Senses into Chinese Dictionaries: A Corpus- 
based Study. Foreign Language and Literature.01, 100-108.

[21] Wienold, G. \& Ulrich Rohmer. (1997). On Implications in Lexicalizations for Dimension Expressions. In Yamanaka, K. \& T. Ohiro(eds.). The Focus of Meaning. Tokyo: Kurosho Press.

[22] Wu, S.X. et, al. (2016). A Corpus Based Comparative Study of English and Chinese Lexical Metaphor. Beijing: China Social Sciences Press.

[23] Xiang, C.D \& Yao, L. (2018). Metaphorical interpretation and Cultural Cognition--Taking Cross-cultural interpretation of polity as an example. Journal of Tianjin Foreign Studies University. 04, 45-55.

[24] Xu, Z.Y. (2014). Metaphor Research in Second Language Acquisition. Beijing: China Social Sciences Press.

[25] Xue, B \& Xiang, M.Y. (2018). A Review on the Knowledge Map of International Corpus-based Metaphor Research in the Past Decade. Journal of PLA University of Foreign Languages. 04,43-51.

[26] Yang, H.Z; Gui, S.C \& Yang D.F.(eds.). (2005). Corpus-based Analysis of Chinese Learner English. Shanghai: Shanghai Foreign Language Education Press.

[27] Yang, N. (2018). The Independent Development of metaphorical competence in Foreign language and the intervention of Learning-type Dictionary: Path and Method. Beijing: Foreign Languages Research, 05, 23-27.

[28] Yu, N. (1998). The Contemporary Theory of Metaphor. Philadelphia: John Benjamin Publishing Company.

[29] Zhao L. (2018). Halliday's Image Schema Basis of Meaning and Metaphorical Clauses. Beijing: Foreign Language Teaching and Research, (04)495-505.

[30] Zhou, J.L. \& Zhang, J.Q. (2003). A Corpus-based Study of Spatial Metaphor. Journal of PLA University of Foreign Languages, $09,27-31$.

Huiying Zhang was born in Hebei, China in 1994. She will receive her Master's degree in TESOL from Beijing International Studies University, China in 2020.

She is a postgraduate student under the instruction of Professor Zhou Weijie in the school of English, Beijing International Studies University, China. Her interests are in corpus linguistic and English teaching, especially in lexical teaching.

Weijie Zhou is currently Associate Professor in Beijing International Studies University. She earned a Master's degree in TESOL in Beijing International Studies University and a Master's degree in Applied Linguistics in Southern Queensland University, Australia.

She has co-authored 6 books and textbooks in Translation and Language teaching. Also she has published more than 10 research papers on language teaching and translation. Her main research interests are Applied Translation and Applied linguistics. 